\title{
JUURNAL.RU
}

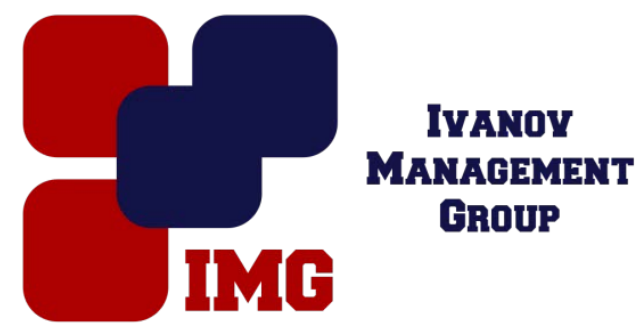

Буткай Д.В., Струков П. С., Суконщиков А.А., Бахтенко Е.А. Вологодский государственный университет

Вологда, Россия

doi: 10.18411/lj-31-01-2017-1-02

idsp 000001:lj-31-01-2017-1-02

\section{Реализация системы семантического поиска на основе метаописаний}

Рассмотримзадачу реализации системы семантического поиска на основе метаописаний. Предложена общая схема системы семантического поиска, которая показана на рис. 1.

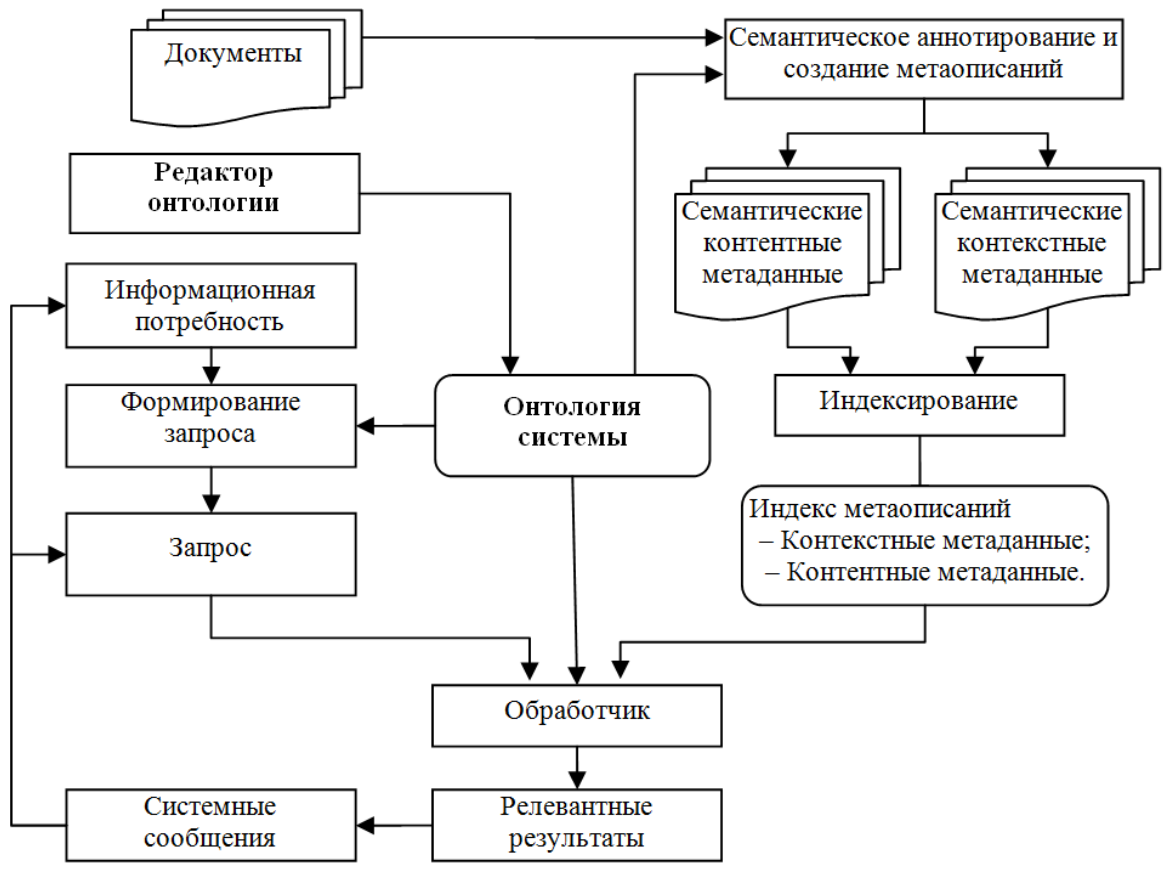

Рис. 1. - Общая схема системы семантического поиска с использованием метаописаний

Базовыми компонентами системы являются онтологии предметных областей. Такие онтологии могут быть созданы ручным путем с помощью редакторов онтологий, например Protégé, либо автоматическим путем.

В отличие от классических систем поиска информации, в предлагаемой схеме при индексировании и формировании запросов создаются метаописанияна 
основе онтологий предметных областей, а обработка запросов выполняется на основе оценок семантической близости между метаописаниями.

Для повышения эффективности создания семантических метаописаний документов нами предложен полуавтоматический метод, согласно которому при создании метаописаний документов система автоматически выделяет элементы онтологии, а человек с помощью редактора выбирает необходимые данные для составления метаописаний. Предложенный метод автоматического выделения элементов онтологии выполняется двумя этапами:поиск меток элементов онтологии в тексте; разрешение многозначности.

Проблема поиска меток элементов онтологии в тексте сформулирована следующим образом: заданы текстовый документД и набор меток элементов онтологии Dic, состоящий из m текстовых меток:

$$
\text { Dic }=\left\{\text { метка }_{1}, \text { метка }_{2}, \ldots, \text { метка }_{m}\right\} .(1)
$$

При этом документД и каждая метка может быть представлена в виде последовательности лемм (лемма - это основная форма слова).

Д = лемма $1 \rightarrow$ лемма $2 \rightarrow \ldots \rightarrow$ леммапи меткаі $=$ лемма $1 \rightarrow \ldots \rightarrow$ леммаk $(\mathrm{i})$,

где $\mathrm{n}$ - количество лемм документаД; $\mathrm{k}(\mathrm{i})$ - количество лемм меткиі.

Каждая ј-я лемма документаД и ј-я лемма i-й метки обозначаются соответственно как Д(j) и меткаі(j).

Требуется найти все пары индексов (i, j), для которых строковое значение является элементом множества меток Diс и ј является максимальным значением среди возможных для фиксированного индекса i.

$$
\text { строка }(i, j, \not)=Д(i) \rightarrow Д(i+1) \rightarrow \ldots \rightarrow Д(j)
$$

Блок-схема предложенного алгоритма решения поставленной задачи показана на рис. 2. Функция $\operatorname{search}(Д, i)$ либо возвращает максимальное значение индекса $j \geq i$, если существуют метки на i-й позиции документаД, либо -1 , если не существуют метки на і-й позиции документа Д.

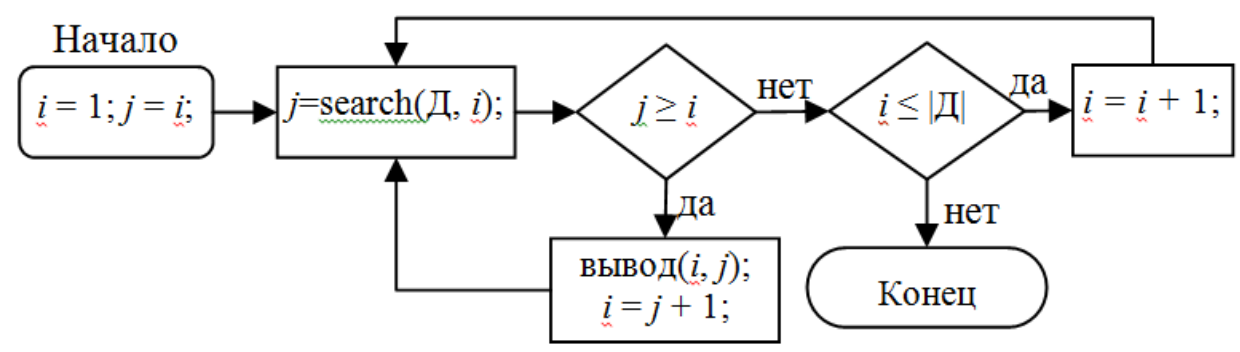

Рис. 2. - Блок-схема алгоритма поиска метокэлементов

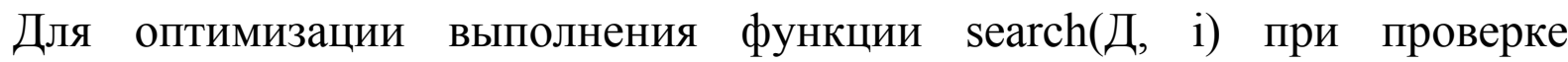
принадлежности метки набору меток предполагается сохранить наборы текстовых меток с использованием структуры данных, представленной на рис. 3 . 


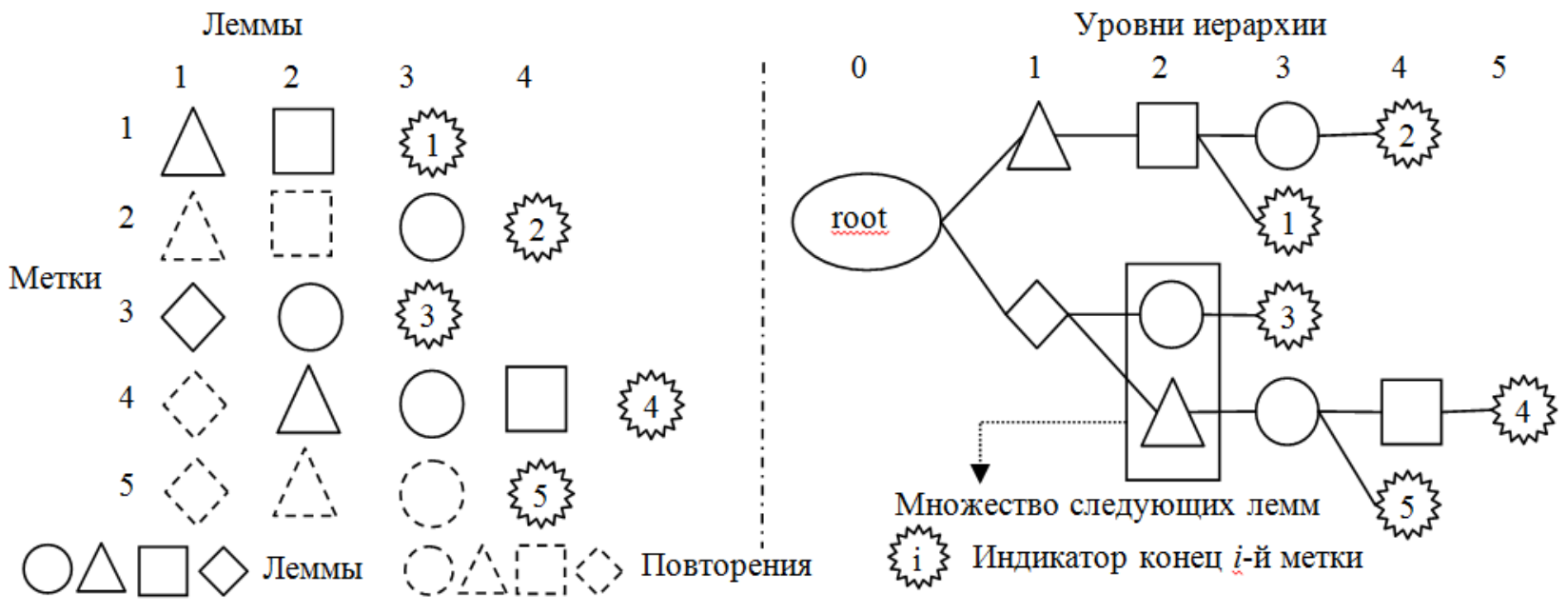

Рис. 3. - Структура данных наборов меток

На основе предложенной структуры данных имеется следующее пошаговое описание алгоритма функции $\operatorname{search}(Д, i)$ :

Шаг 1. Началоалгоритма, j = i; jmax = -1 ; pointer = root;

Шаг2.pointer $=$ pointer.next(Д(j)) - переход на следующий узел, содержащий леммуД(j).

Если существует узел $p \in$ pointer.next, содержащий уникальный идентификатор, то:

обновить значение $\mathrm{jmax}=\mathrm{j} ; \mathrm{j}=\mathrm{j}+1$; повторить шаг 2 ;

иначе если pointer != null, то:

$\mathrm{j}=\mathrm{j}+1$; повторить шаг 2 ;

иначе на шаг 3.

Шаг 3: вывод jmax; конец алгоритма.

Следующим шагом после поиска меток является разрешение многозначности, т. е. определение подходящего элемента онтологии для каждой метки. Для этой цели нами были рассмотрены два существующих метода разрешения многозначности: разрешение многозначности на основе оценок семантической близости; разрешение многозначности на основе степени популярности.

Для обеспечения функционирования предложенной системы семантического поиска требуется создание индексов, показанных на рис. 4. 


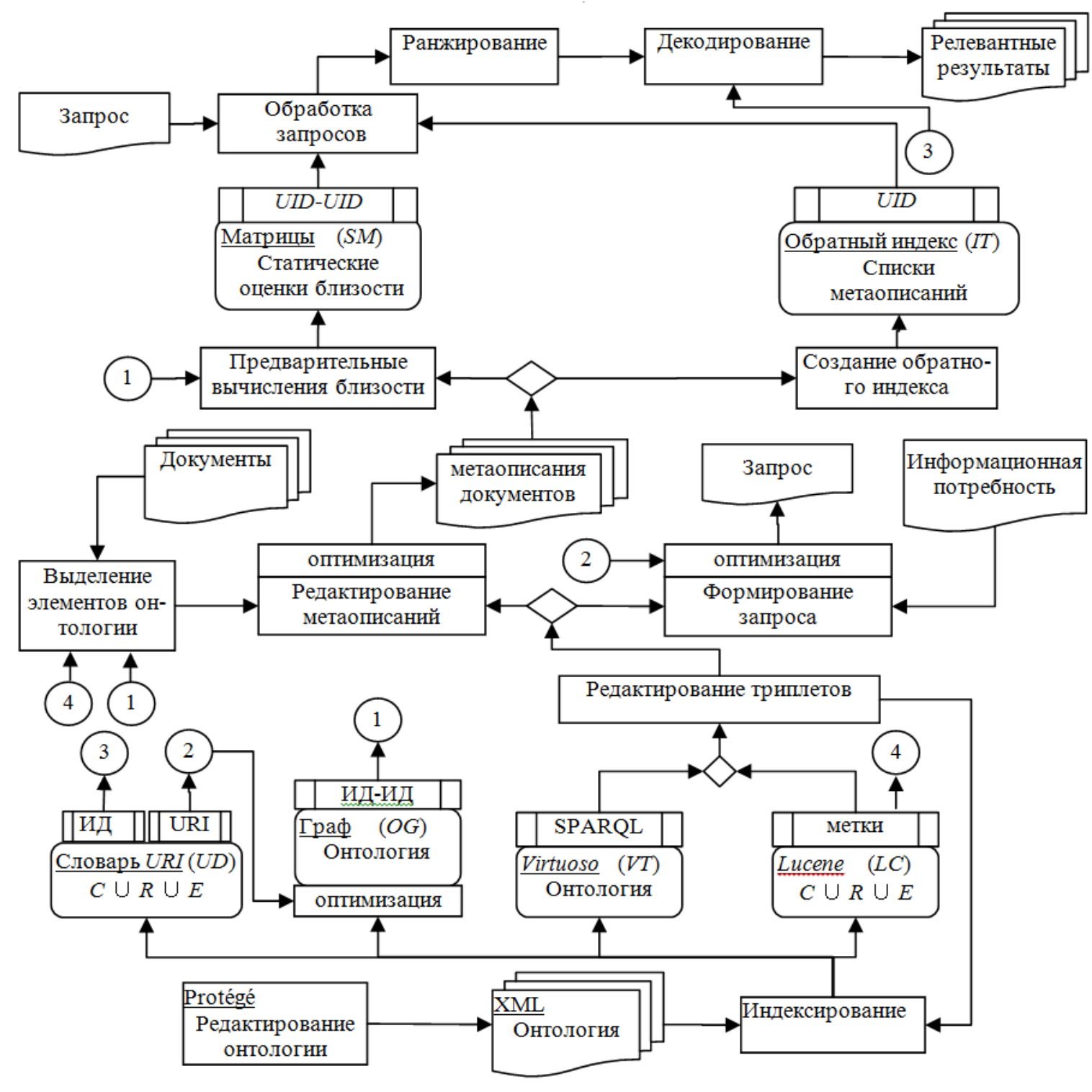

Рис. 4. - Структура индексов предложенной системысемантического поиска

Как показано на рис. 4, при индексировании онтологий создаются индексы $\mathrm{UD}, \mathrm{OG}, \mathrm{VT}$ и LC, а при индексировании метаописаний электронных ресурсов создаются индексы SM и IT. Индексы VT и LC используются в основном функциями формирования триплетов для выборов элементов онтологии, а индекс OG используется для нахождения путей между элементами онтологии, на основе которых вычисляются их близости.

В индексе SM сохраняются оценки близости между элементами онтологии для цели исключения их повторных вычислений, а в индексе IT сохраняются инвертированные списки метаописаний для выполнения их фильтрации при обработке запросов.

На основе индекса UD разработан метод оптимизации хранения коллекции триплетов с использованием числовых идентификаторов, предназначенный для сокращения требуемых объемов памяти при хранении наборов триплетов. 
Основная идея предложенного метода заключается в замене каждого URIидентификатора на уникальный числовой идентификатор, который затем может быть преобразован обратно в строку URI. Сопоставления между URIидентификаторами и числовыми идентификаторами сохраняются в словаре URIидентификаторов, структура которого показана на рис. 5.

Словарь $U R I$-идентификаторов без сжатия

после сжатия

\begin{tabular}{|c|c|c|c|c|c|}
\hline i & строки URI в явном виде & $\mathrm{d}[\mathrm{i}]$ & $\mathrm{p}[\mathrm{i}]$ & ид & строки URI после сжатия \\
\hline 1 & http://dbpedia.org/resource/Alexei_Gusarov & 0 & 1 & 1 & http://dbpedia.org/resource/ Alexei Gusarov \\
\hline 2 & http://dbpedia.org/resource/James_R._Russell & 28 & 1 & 2 & James $R$. Russell \\
\hline 3 & http://dbpedia.org/resource/Martina_Hellmann & 28 & 1 & 3 & Martina Hellmann \\
\hline
\end{tabular}

Рuc. 5. Структура словаря URI с использованием метода сжатия

C целью сокращения требуемого объема оперативной памяти для хранения словаря URI-идентификаторовразработан специальный метод сжатия без потери для набора строк URI и соответствующий метод декодирования. Как показано на рис. 5, с использованием разработанного метода сжатия потребуется меньше памяти для сохранения строки URI за счет удаления их общих фрагментов (например, если все строки URI начинаются c http://.., data://... и т.п.). 